\title{
Serum Neopterin Is Not Increased in Obese Juveniles
}

\author{
Harald Mangge, ${ }^{1}$ Florian Freytag, ${ }^{1}$ Gunter Almer, ${ }^{1}$ Daniel Weghuber, ${ }^{2}$ \\ Carmen Bauer-Denk, ${ }^{1}$ and Dietmar Fuchs ${ }^{3}$ \\ ${ }^{1}$ Clinical Institute of Medical and Chemical Laboratory Diagnostics, Medical University of Graz, 8036 Graz, Austria \\ ${ }^{2}$ Department of Pediatrics, University Hospital of Salzburg, 5020 Salzburg, Austria \\ ${ }^{3}$ Division of Biological Chemistry, Biocenter, Innsbruck Medical University, 6020 Innsbruck, Austria
}

Correspondence should be addressed to Harald Mangge, harald.mangge@meduni-graz.at

Received 11 June 2010; Accepted 22 December 2010

Academic Editor: A. Halpern

Copyright (C) 2011 Harald Mangge et al. This is an open access article distributed under the Creative Commons Attribution License, which permits unrestricted use, distribution, and reproduction in any medium, provided the original work is properly cited.

\begin{abstract}
Objective. Cardiovascular disease is associated with inflammation and immune activation, concentrations of immune activation markers like neopterin predict outcome in adults. Methods. Serum neopterin concentrations and early metabolic and preatherosclerotic symptoms were analyzed in 295 obese juveniles and 101 normal weight controls of similar age. Additionally, the influence of a 12 months weight reduction program on neopterin levels was investigated in 31 obese juveniles. Results. Intimamedia thickness of common carotid arteries (IMT) and the concentrations of C-reactive protein (CRP) were increased in the obese juveniles $(P<.001)$. Also triglycerides, oxidized LDL, fasted insulin levels, HOMA-index, leptin, liver transaminases and uric acid were increased compared to the controls. However, serum neopterin was decreased in the obese versus non-obese juveniles $(P<.03)$. The intervention consisting of regular sports, nutritional devices, and a psychologic attendance led after 12 months to an increase of neopterin concentration $(P<.05$; paired test). Conclusions. Neopterin concentrations in juvenile obesity behaved considerably different from what was demonstrated in adults, levels did not correlate with metabolic and pre-atherosclerotic symptoms found in early phases although early vascular burden and chronic low grade inflammation was indicated by increased IMT and CRP. Neopterin concentrations increased after a 12 months intervention program.
\end{abstract}

\section{Introduction}

Nowadays, the role of inflammation and immune activation in atherogenesis is well established [1], and the activation of macrophages appears to be of critical relevance within this process $[1,2]$. Cardiovascular disease $(\mathrm{CVD})$ is also associated with increased neopterin concentrations [3-10], and predictive information is provided by neopterin in patients at risk of myocardial infarction [11-16]. A recent study in 2312 patients undergoing angiography demonstrated that neopterin is an independent predictor of total and cardiovascular mortality [17]. Likewise, in patients with diabetes, both neopterin and C-reactive protein (CRP) were independent predictors of fatal ischemic heart disease [18].

Neopterin is produced by human monocyte-derived macrophages and dendritic cells preferentially upon stimulation with Th1-type cytokine interferon- $\gamma($ IFN- $\gamma)[19,20]$.
Thus, neopterin concentrations reflect activation of the Th1type immune response [21]. In humans, increased neopterin concentrations are detected in patients with virus infections including human immunodeficiency virus, infections by intracellular bacteria and parasites, autoimmune diseases, malignant tumors, and during rejection episodes in allograft recipients [21-24].

In foregoing studies, we detected an increased carotid intima-media thickness (IMT) indicating preatherosclerosis paralleled by a subclinical inflammation in obese juveniles aged around 13 years [25]. Additionally, we showed an influence of subcutaneous adipose tissue topography on total adiponectin and adiponectin subfraction serum levels in obese juveniles and provided the first evidence that incipient atherosclerosis yet found in adolescents by an increased IMT is associated with a disturbed oligomerisation from lowmolecular to high-molecular-weight adiponectin $[26,27]$. 
In a group of otherwise healthy adults, a positive correlation was observed between neopterin concentrations and body mass index [28]. From the data obtained in adults, it appears that neopterin serum levels are associated with chronic subclinical inflammation and preatherosclerotic symptoms in early phases of obesity in a particular manner. In this study, we analysed serum concentrations of neopterin with carotid IMT, and cardiovascular risk factors in a cohort of 295 obese juveniles and adolescents compared to 101 normal weighted, healthy controls of the same age and gender distribution. Further, we investigated the influence of a weight reduction program performed for 12 months in a subgroup of 40 obese juveniles on neopterin serum concentrations.

\section{Materials and Methods}

2.1. Patients. Study participants were from the STYrian Juvenile Obesity Study (STYJOBS), which is designed to investigate early stages of atherosclerosis and metabolic disorders in obese juveniles. STYJOBS is registered at http://clinicaltrials.gov/ (Identifier NCT00482924), where detailed information of the study is available. The inclusion criterium for the obese probands was body mass index $(\mathrm{BMI})>97$ th percentile if under 18 years of age, BMI $>30 \mathrm{~kg} / \mathrm{m}^{2}$ if over 18 years of age. Exclusion criteria were endocrine diseases (e.g., hypothyroidism), infectious, inflammatory, or any other chronic diseases. Controls were healthy juvenile volunteers recruited from the Department of Pediatric Surgery, where they underwent minor elective surgery (e.g., herniotomia). Fasted blood samples were collected prior to anaesthesia and surgery. All controls had to be normal weight, free of infectious, and endocrine or any other diseases. 295 juveniles (mean age $12.9 \pm 3.3$ SD years) and 101 normal weight healthy controls of similar age and gender distribution were investigated.

Out of this cohort, 40 juveniles with BMI > 97th percentile (male 17 , female 23 , mean age $13.6+2.3$ years $(\mathrm{SD})$ ) underwent a 12-month weight reduction program during the year 2006. The intervention schedule encompassed regular sports ( 2 times per week, one hour, 12 months), nutritional devices $(1 \mathrm{x} /$ week, 1 st three months, $1 \mathrm{x} / 2$ weeks, 2 nd three months, $1 \mathrm{x} / 4$ weeks within the remaining 6 months), and a psychologic attendance. Thirtyone obese juveniles (male 13, female 18) who fully completed this program were included in the analysis of the neopterin kinetics during intervention.

The study was approved by the Ethical Committee of the Medical University of Graz. Blood collection and ultrasonography were performed after written informed consent was given by the probands if aged over 18 years, or by their parents, if they were under 18 years old. At the time of blood collection, the probands were fasting. Venous puncture was performed in a standard procedure (cubital vein approach with butterfly); blood samples were immediately centrifuged at $3500 \mathrm{rpm}$ at ambient temperature and stored at $-80^{\circ} \mathrm{C}$ until analysis.
2.2. Carotid Artery Ultrasound. The ultrasound protocol involved scanning of the bulbous near the common carotid artery on both sides with a 12 -to-5-MHz broad-band linear transducer on an HDI 5000 (ATL, Bothell, Washington, DC, USA). All scans were performed by the same investigator. Longitudinal images directed through the center of the artery were taken at each vessel site. Measurements were made from stored digital images by an experienced reader. The carotid IMT was assessed at the far wall as the distance between the interface of the lumen and intima, and the interface between the media and adventitia. The maximal IMT was recorded at each of the vessel segments and averaged for the left and right sides. The lumen diameter was calculated as the interadventitial diameter minus twice the maximum far wall IMT. All diameters were measured during diastole to avoid image blurring due to systolic arterial wall motion and to minimize the influence of blood pressure [29].

2.3. Lipometry. Measurements of total percentual body fat were performed as described elsewhere by means of a lipometer, a patented optical device (EU Pat.Nr. 0516251) [30].

2.4. Laboratory Analysis. Neopterin concentrations were determined by enzyme-linked immunosorbant assay (ELISA) (BRAHMS Diagnostica, Hennigsdorf, Germany). Total adiponectin, leptin, and resistin were determined by ELISA (Biovendor Laboratory Medicine Inc., Brno, Czech Republic) according to manufacturer's instructions. Intra- and interassay coefficients of variation for all ELISAs in our study were below 10 percent. Cholesterol and triglycerides were measured by means of ECLIA (Electro Chemi Luminiscence Assay) on an Elecsys 2010 analyser (Roche Diagnostics Mannheim, Germany), and lipoproteins were separated by a combined ultracentrifugation-precipitation method $(\beta$ quantification). Blood lipids inclusive of fatty acids were analysed as outlined elsewhere [31]. Oxidized low-dense lipoprotein (oxLDL) was measured by a commercially available ELISA (Mercodia oxidized LDL Competitive ELISA, 754 50 Uppsala, Sweden). Concentrations of CRP were analysed with a highly sensitive particle-enhanced immunoturbidimetric assay (Tina-quant C-reactive protein latex ultrasensitive assay, Roche diagnostics). Plasma insulin was measured by ELISA (Mercodia, Uppsala, Sweden) and plasma glucose was measured by the glucose hexokinase method on a Hitachi 917 chemical analyser. HOMA-IR (homeostatic model assessment-insulin resistance) was calculated as the product of the fasting plasma insulin value (in $\mu \mathrm{U} / \mathrm{ml}$ ) and the fasting plasma glucose value $(\mathrm{mm} / \mathrm{L})$, divided by 22.5 [27]. Liver transaminases AST/GOT, ALT/GPT, $\boldsymbol{\gamma}$ GT, creatinine, and uric acid were measured by routine laboratory methods on a Hitachi 917 chemical analyser.

2.5. Statistic Analysis. Statistical analysis was performed by SPSS version 14. Kolmogorov-Smirnov test was used to examine for normal distribution. Means were compared by a two-tailed unpaired sample $t$-test or by Mann-Whitney 
Test, depending on the distribution of the data. A value of $P<.05$ was considered statistically significant. Least squares regression analysis was performed to test the correlation between variables according to Pearson. Significance of correlation was determined by univariate ANOVA and subsequent multiple comparison analysis. Bonferroni correction was used for multiple comparisons after ANOVA. To exclude a dependence of the resulting correlations from other variables, the correlations were adjusted by partial correlations. Stepwise multiple regression analysis was applied to investigate correlations between neopterin as dependent variable and different metabolic and vascular measures as independent variables.

\section{Results}

The clinical and laboratory characteristics of the study probands are summarized in Table 1. Obese juveniles had a significantly increased IMT $(P<.001)$ and elevated CRP, $(P<.001$, see also Figure 1$)$ indicating early stages of atherosclerosis and chronic low-grade inflammation (Table 1). Oxidized LDL, fasted insulin, HOMAindex, resistin, and systolic-, diastolic-blood pressure were significantly increased, and HDL cholesterol significantly decreased in the obese cohort (Table 1). Compared to the controls, obese juveniles had significantly lower neopterin serum concentrations (Table 1 and Figure 1). Neopterin concentrations did not correlate with percentual body fat or BMI-SDS values in the whole study cohort and were independent from any other variable investigated in this study and listed in Table 1 (data not shown).

After the 12-month weight reduction program consisting of regular sports, nutritional devices, and a psychologic attendance, the total percentual total body fat, oxLDL, and resistin serum concentrations decreased significantly whereas the HDL-cholesterol levels were significantly increased (Table 2). CRP concentrations increased upon intervention as compared with baseline, but the difference was not significant. Fasted blood glucose, insulin levels, IMT of common carotid arteries, and systolic and diastolic blood pressure did not differ significantly from baseline (details not shown). After the 12-month intervention program, neopterin concentrations increased and the mean value became higher than baseline (Table 2). This increase of neopterin concentrations did not correlate with the decrease of the BMI-SDS.

\section{Discussion}

Our study shows that serum neopterin concentrations are not increased in obese adolescents compared to nonobese controls, rather a trend to subnormal levels was observed. Although neopterin concentrations were lowered only by approximately $10 \%$ compared to the nonobese controls, the difference was significant. This result is astonishing compared to results obtained in several studies performed earlier by various research groups in adults: adults with high risk for atherosclerosis presented with slightly but significantly

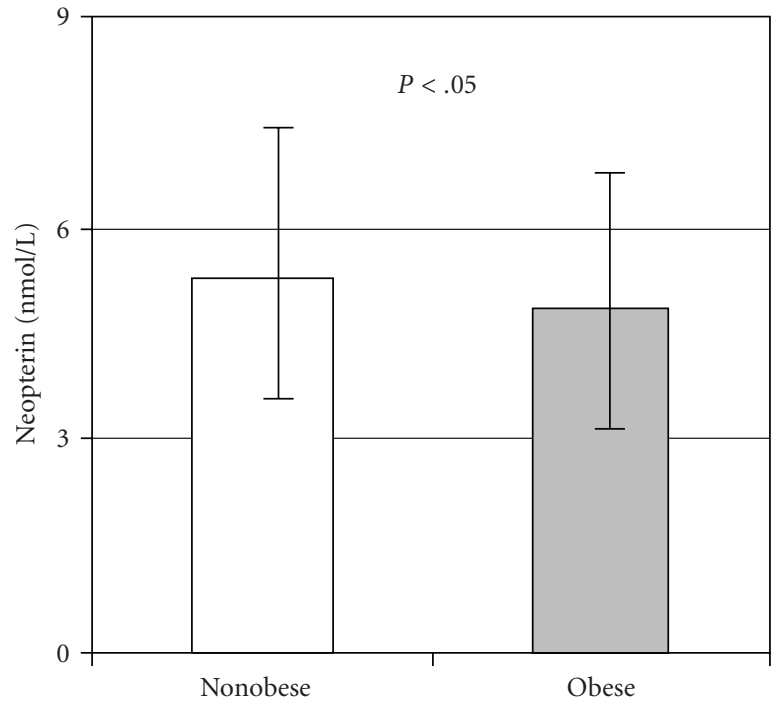

(a)

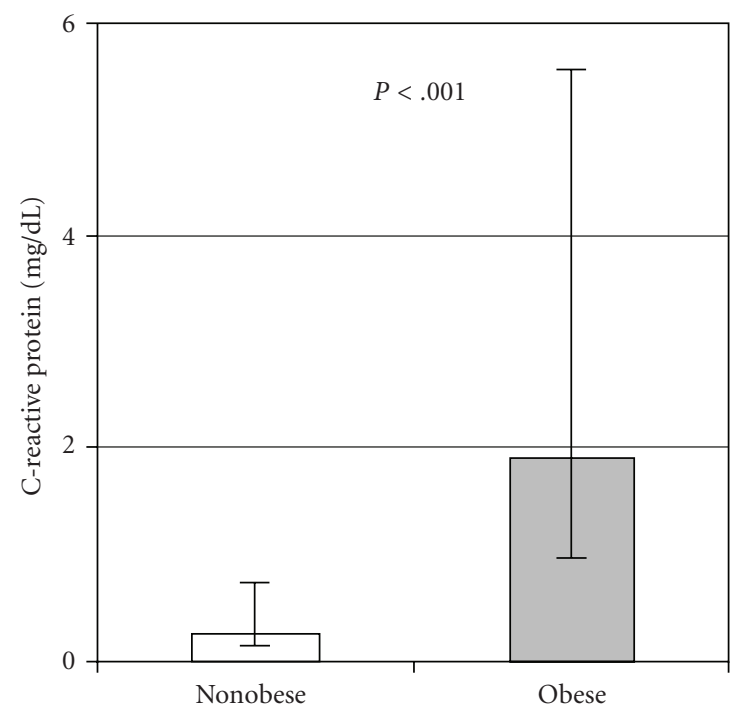

(b)

FIGURE 1: Serum concentrations of neopterin and C-reactive protein in obese and nonobese adolescents. Neopterin concentrations are given as mean values (boxes) and S.E.M. (bars) and because of nonnormal distribution C-reactive protein is given as medians (boxes) and 25-75th percentiles (bars).

increased serum neopterin concentrations compared to lowrisk persons [11-18, 32]. Myocardial infarction (MI) is usually accompanied by a transient increase of neopterin [33]. Although only moderately elevated compared to other clinical conditions like acute virus infections or cancer, neopterin levels showed a predictive potential for future clinical events in symptomatic and stable CVD patients $[12,15,17,18,34]$. This predictive value of neopterin was found to be independent from other biomarkers such as CRP $[16-18,32]$. The ability to enhance oxidative stress and to induce proinflammatory cytokine cascades [33, 35 ] via induction of nuclear factor $\kappa \mathrm{B}$ suggests neopterin 
TABLE 1: Characteristics of the study subjects.

\begin{tabular}{lcc}
\hline Variable & Controls $(n=101)$ & Obese adolescents $(n=295)$ \\
\hline Age (Years) & $12.9 \pm 2.7(4-18)$ & $12.4 \pm 2.9(4-18)$ \\
Body Length $(\mathrm{m})$ & $1.56 \pm 0.14$ & $1.56 \pm 0.14$ \\
Body Weight $(\mathrm{kg})$ & $47.2 \pm 13.4$ & $74.5 \pm 24.2^{* *}$ \\
BMI $\left(\mathrm{kg} / \mathrm{m}^{2}\right)$ & $18.7(16.9-20.4)$ & $28.9(26.2-32.4)^{+++}$ \\
BMI-SDS & $0.21(-0.7-0.8)$ & $5.4(4.0-7.2)^{+++}$ \\
Carotid IMT (cm) & $0.052 \pm 0.009$ & $0.070 \pm 0.09^{* * *}$ \\
Neopterin (nmol/L) & $5.3 \pm 1.9$ & $4.9 \pm 1.8^{*}$ \\
C-Reactive protein (mg/dl) & $0.26(0.17-0.84)$ & $1.9(0.9-3.8)^{+++}$ \\
HDL-cholesterol (mg/dl) & $48.4 \pm 12.0$ & $41.9 \pm 11.2^{* * *}$ \\
Oxidized LDL (mg/dl) & $35.1 \pm 15.3$ & $44.3 \pm 16.9^{* * *}$ \\
Insulin (uE/ml) & $7.9(5.1-12.7)$ & $15.4(8.8-29.1)^{+++}$ \\
HOMA-IR & $1.7(1.0-2.7)$ & $3.5(1.8-6.9)^{+++}$ \\
Resistin (ng/ml) & $3.7 \pm 1.5$ & $4.3 \pm 1.6^{* *}$ \\
SBP (mmHg) & $115 \pm 4.9$ & $125 \pm 15.5^{* * *}$ \\
DBP (mmHg) & $63.1 \pm 9$ & $68 \pm 8.8^{* * *}$ \\
\hline
\end{tabular}

Results are expressed as mean \pm S.D. analysed by Student's $t$-test $\left({ }^{*} P<.05,{ }^{* *} P<.01,{ }^{* * *} P<.001\right)$ or as median $(25$ th-75th percentile) and Mann Whitney $U$ test $\left({ }^{+} P<.05,{ }^{++} P<.01,{ }^{+++} P<.001\right)$ depending on the distribution of data.

BMI, body mass index; BMI-SDS, BMI-standard deviation score; HOMA-IR, homeostatic model assessment-insulin resistance; SBP, systolic blood pressure; DBP, diastolic blood pressure; carotid IMT, carotid intima-media thickness.

TABLE 2: Neopterin, body fat, HDL cholesterol, resistin, oxLDL, and C-reactive protein concentrations (mean \pm S.D.) before and after 12 months of intervention therapy.

\begin{tabular}{lcc}
\hline & Start of intervention & End of intervention (12 months) \\
\hline$n$ & 31 (male 13, female 18) & 31 \\
Neopterin (nmol/L) & $4.4 \pm 0.38$ & $5.0 \pm 1.7^{*}$ \\
Body fat (\%) & $36.8 \pm 7.0$ & $29.5 \pm 5.1^{* * *}$ \\
HDL cholesterol (mg/dl) & $42.4 \pm 7.6$ & $47.2 \pm 9.3^{*}$ \\
Resistin $(\mathrm{ng} / \mathrm{ml})$ & $4.7 \pm 1.6$ & $3.9 \pm 0.9^{*}$ \\
Oxidized LDL $(\mathrm{mg} / \mathrm{dl})$ & $59.7 \pm 10.8$ & $53.1 \pm 16.3^{*}$ \\
C-reactive protein $(\mathrm{mg} / \mathrm{dl})$ & $2.8 \pm 2.6$ & $3.2 \pm 3.2$ \\
\hline
\end{tabular}

${ }^{*} P<.05,{ }^{* *} P<.01,{ }^{* * *} P<.001$ (paired $t$-test).

to act as a proinflammatory biomarker in atherogenesis [36]. Neopterin appears to play an important role in the pathogenesis of CVD. However, to our surprise this seems to be true only in adults but not in juvenile obesity. There is already information available from adults that CAD activity more than the size of lesions is important for the elevation of neopterin, but in contrast, despite detectable CAD activity in juvenile obesity, neopterin concentrations tended to even lower levels than in nonobese controls of similar age.

Increased neopterin concentrations are characteristic for an activated Th1-type immune response [22]. Accordingly, the decreased or low-neopterin concentrations found in our study cohort of obese adolescents even if rather small in absolute terms might indicate a suppressed Th1type immunity and thus lowered IFN- $\gamma$ production [37]. In fact, only the neopterin concentrations found in the obese groups were lower than normal, the mean value of neopterin concentrations measured in the nonobese control population $(5.3 \pm 1.9 \mathrm{nmol} / \mathrm{L}$; Table 1$)$ was almost identical with the one found in larger populations of adult blood donors and healthy controls as $5.3 \pm 2.7 \mathrm{nmol} / \mathrm{L}[21,22$, 38]. At present, increased neopterin concentrations are well established in patients with several diseases which are known to be associated with immune activation such as virus infections, autoimmune syndromes, and various types of cancer $[21,22]$. However, subnormal or suppressed neopterin release in clinical disease is rather rare. Only from in vitro studies, it is known that several antioxidant compounds are able to slowdown neopterin production in peripheral blood mononuclear cells. Among them are antioxidant food supplements like preservatives and colorants, but also high dose of antioxidant vitamins $\mathrm{C}$ and $\mathrm{E}$ suppresses Th1-type cytokines and inducees a Th2-type immunity pattern in vitro [39].

Whereas antioxidant compounds were observed to suppress Th1-type immunity and neopterin production in vitro, physical exercise and sports like long-distance running and hiking were found to induce a transient increase of neopterin concentrations $[40,41]$. An increase of neopterin concentrations was also observed in a rather small subgroup 
of our patients who attended an intervention therapy during one year that comprised improved dietary habits and the increase of exercise. This increase of neopterin indicates a proinflammatory response which is induced by exercise and corresponds nicely to the above-mentioned earlier findings in healthy individuals. Thus, the most direct and consistent interpretation would be that physical exercise was responsible for the increase of neopterin concentrations.

One may conclude that low or even subnormal neopterin concentrations may relate to a low incidence of physical activities in obese juveniles. Moreover, in diabetes patients, antioxidants have been recently found to prevent the healthpromoting effects of physical exercise [42]. In conclusion, low frequency of physical exercise and sports as well as antioxidant food supplements could contribute to the low neopterin levels in obese juveniles, and it may contribute to weight gain. However, this is merely speculation and still the increase of body weight and the decrease of neopterin might well develop in parallel and are not necessarily linked directly.

Unfortunately, the findings of the intervention program appear somewhat hampered by the fact that those individuals who were willing to enter this program presented with baseline levels somewhat different from the whole study group, for example, neopterin concentrations were slightly lower and resistin and CRP levels were slightly but not significantly higher. The intervention program increased neopterin concentrations, but the average neopterin level reached was only slightly higher as compared to the whole group without any special sports activities. Still the difference of neopterin concentrations before and after intervention was significant when paired samples of the subgroup were compared. Certainly a larger study will be necessary to adequately address this issue, but it is not easy to get.

According to the findings on neopterin concentrations, obesity in adolescents might be classified as a condition linked with Th2-type immunity. It will be interesting to investigate in future whether the balance between Th1- and Th2-type immunity is essentially involved in the observed neopterin kinetics and in obesity development. It can be speculated that the early low-grade inflammation, as found in our obese juveniles in context with an already increased IMT, may be a more Th2-driven process, whereas the advanced atherosclerosis of adulthood is associated with a stronger Th1-type immune activation. There might be a role of mast cells which are known to be strongly engaged in the allergic response but also have the potential to promote atherosclerosis by releasing proinflammatory cytokines which could become relevant with older age [43].

In conclusion, the finding of low-serum neopterin concentrations in obese juveniles forces one to consider that the pathogenesis of cardiovascular disease development in children and juveniles may differ from adults. Further studies should help to better define age-dependent differences and to define optimized preventive strategies, not only the increase of physical exercise and sports but also avoidance of antioxidant food supplements such as preservatives or extra vitamins might be of benefit.

\section{Abbreviations}

$\begin{array}{ll}\text { BMI: } & \text { Body mass index } \\ \text { CVD: } & \text { Cardiovascular disease } \\ \text { CRP: } & \text { C-reactive protein } \\ \text { ELISA: } & \text { Enzyme-linked immunosorbant assay } \\ \text { HDL: } & \text { High-density lipoprotein } \\ \text { HOMA-IR: } & \text { Homeostatic model assessment-insulin } \\ & \text { resistance } \\ \text { IFN- } \gamma: & \text { Interferon- } \gamma \\ \text { IMT: } & \text { Carotid intima-media thickness } \\ \text { LDL: } & \text { Low-density lipoprotein } \\ \text { MI: } & \text { Myocardial infarction } \\ \text { oxHDL: } & \text { Oxidized high-density lipoprotein } \\ \text { oxLDL: } & \text { Oxidized low-density lipoprotein } \\ \text { STYJOBS: } & \text { STYrian Juvenile Obesity Study. }\end{array}$

\section{Acknowledgment}

The authors thank Miss Astrid Haara and Miss Kerstin Hingerl for excellent technical assistance.

\section{References}

[1] G. K. Hansson, "Mechanisms of disease: inflammation, atherosclerosis, and coronary artery disease," The New England Journal of Medicine, vol. 352, no. 16, pp. 1685-1626, 2005.

[2] S. Vonhof, B. Brost, M. Stille-Siegener, I. M. Grumbach, H. Kreuzer, and H. R. Figulla, "Monocyte activation in congestive heart failure due to coronary artery disease and idiopathic dilated cardiomyopathy," International Journal of Cardiology, vol. 63, no. 3, pp. 237-244, 1998.

[3] F. Tatzber, H. Rabl, K. Koriska et al., "Elevated serum neopterin levels in atherosclerosis," Atherosclerosis, vol. 89, no. 2-3, pp. 203-208, 1991.

[4] G. Weiss, J. Willeit, S. Kiechl et al., "Increased concentrations of neopterin in carotid atherosclerosis," Atherosclerosis, vol. 106, no. 2, pp. 263-271, 1994.

[5] M. Schumacher, G. Halwachs, F. Tatzber et al., "Increased neopterin in patients with chronic and acute coronary syndromes," Journal of the American College of Cardiology, vol. 30, no. 3, pp. 703-707, 1997.

[6] E. P. Gurfinkel, B. M. Scirica, G. Bozovich, A. Macchia, E. Manos, and B. Mautner, "Serum neopterin levels and the angiographic extent of coronary arterial narrowing in unstable angina pectoris and in non-Q-wave acute myocardial infarction," American Journal of Cardiology, vol. 83, no. 4, pp. 515-518, 1999.

[7] S. Kiechl, E. Lorenz, M. Reindl et al., "Toll-like receptor 4 polymorphisms and atherogenesis," The New England Journal of Medicine, vol. 347, no. 3, pp. 185-192, 2002.

[8] E. Zouridakis, P. Avanzas, R. Arroyo-Espliguero, S. Fredericks, and J. C. Kaski, "Markers of inflammation and rapid coronary artery disease progression in patients with stable angina pectoris," Circulation, vol. 110, no. 13, pp. 1747-1753, 2004.

[9] P. Avanzas, R. Arroyo-Espliguero, J. Quiles, D. Roy, and J. C. Kaski, "Elevated serum neopterin predicts future adverse cardiac events in patients with chronic stable angina pectoris," European Heart Journal, vol. 26, no. 5, pp. 457-463, 2005.

[10] A. Dominguez-Rodriguez, P. Abreu-Gonzalez, and M. GarciaGonzalez, "Usefulness of neopterin levels and left ventricular function for risk assessment in survivors of acute myocardial 
infarction," International Journal of Cardiology, vol. 111, no. 2, pp. 318-320, 2006.

[11] D. T. Johnston, M. Gagos, N. Raio et al., "Alterations in serum neopterin correlate with thrombolysis in myocardial infarction risk scores in acute coronary syndromes," Coronary Artery Disease, vol. 17, no. 6, pp. 511-516, 2006.

[12] K. K. Ray, D. A. Morrow, M. S. Sabatine et al., "Long-term prognostic value of neopterin: a novel marker of monocyte activation in patients with acute coronary syndrome," Circulation, vol. 115, no. 24, pp. 3071-3078, 2007.

[13] T. Adachi, T. Naruko, A. Itoh et al., "Neopterin is associated with plaque inflammation and destabilisation in human coronary atherosclerotic lesions," Heart, vol. 93, no. 12, pp. 1537-1541, 2007.

[14] J. C. Kaski, L. Consuegra-Sanchez, D. J. Fernandez-Berges et al., "Elevated serum neopterin levels and adverse cardiac events at 6 months follow-up in Mediterranean patients with non-ST-segment elevation acute coronary syndrome," Atherosclerosis, vol. 201, no. 1, pp. 176-183, 2008.

[15] H. F. Alber, C. Duftner, M. Wanitschek et al., "Neopterin, $\mathrm{CD} 4{ }^{+} \mathrm{CD} 28^{-}$lymphocytes and the extent and severity of coronary artery disease," International Journal of Cardiology, vol. 135, no. 1, pp. 27-35, 2009.

[16] V. Videm, R. Wiseth, S. Gunnes, H. O. Madsen, and P. Garred, "Multiple inflammatory markers in patients with significant coronary artery disease," International Journal of Cardiology, vol. 118, no. 1, pp. 81-87, 2007.

[17] T. B. Grammer, D. Fuchs, B. O. Boehm, B. R. Winkelmann, and W. Maerz, "Neopterin as a predictor of total and cardiovascular mortality in individuals undergoing angiography in the Ludwigshafen Risk and Cardiovascular Health study," Clinical Chemistry, vol. 55, no. 6, pp. 1135-1146, 2009.

[18] I. T. Vengen, A. C. Dale, R. Wiseth, K. Midthjell, and V. Videm, "Neopterin predicts the risk for fatal ischemic heart disease in type 2 diabetes mellitus. Long-term follow-up of the HUNT 1 study," Atherosclerosis, vol. 207, no. 1, pp. 239-244, 2009.

[19] C. Huber, J. R. Batchelor, and D. Fuchs, "Immune response-associated production of neopterin. Release from macrophages primarily under control of interferon-gamma," Journal of Experimental Medicine, vol. 160, no. 1, pp. 310-316, 1984.

[20] B. Wirleitner, D. Reider, S. Ebner et al., "Monocyte-derived dendritic cells release neopterin," Journal of Leukocyte Biology, vol. 72, no. 6, pp. 1148-1153, 2002.

[21] C. Murr, B. Widner, B. Wirleitner, and D. Fuchs, "Neopterin as a marker for immune system activation," Current Drug Metabolism, vol. 3, no. 2, pp. 175-187, 2002.

[22] D. Fuchs, G. Weiss, G. Reibnegger, and H. Wachter, "The role of neopterin as a monitor of cellular immune activation in transplantation, inflammatory, infectious, and malignant diseases," Critical Reviews in Clinical Laboratory Sciences, vol. 29, no. 3-4, pp. 307-341, 1992.

[23] C. Murr, A. Bergant, M. Widschwendter, K. Heim, H. Schröcksnadel, and D. Fuchs, "Neopterin is an independent prognostic variable in females with breast cancer," Clinical Chemistry, vol. 45, no. 11, pp. 1998-2004, 1999.

[24] G. Reibnegger, C. Aichberger, D. Fuchs et al., "Posttransplant neopterin excretion in renal allograft recipients-a reliable diagnostic aid for acute rejection and a predictive marker of long-term graft survival," Transplantation, vol. 52, no. 1, pp. 58-63, 1991.

[25] H. Mangge, K. Schauenstein, L. Stroedter, A. Griesl, W. Maerz, and M. Borkenstein, "Low grade inflammation in juvenile obesity and type 1 diabetes associated with early signs of atherosclerosis," Experimental and Clinical Endocrinology and Diabetes, vol. 112, no. 7, pp. 378-382, 2004.

[26] S. Pilz, R. Horejsi, R. Möller et al., "Early atherosclerosis in obese juveniles is associated with low serum levels of adiponectin," Journal of Clinical Endocrinology and Metabolism, vol. 90, no. 8, pp. 4792-4796, 2005.

[27] H. Mangge, G. Almer, S. Haj-Yahya et al., "Nuchal thickness of subcutaneous adipose tissue is tightly associated with an increased LMW/total adiponectin ratio in obese juveniles," Atherosclerosis, vol. 203, no. 1, pp. 277-283, 2009.

[28] M. Ledochowski, C. Murr, B. Widner, and D. Fuchs, "Association between insulin resistance, body mass and neopterin concentrations," Clinica Chimica Acta, vol. 282, no. 1-2, pp. 115-123, 1999.

[29] S. Kiechl and J. Willeit, "The natural course of atherosclerosis: part II: vascular remodeling," Arteriosclerosis, Thrombosis, and Vascular Biology, vol. 19, no. 6, pp. 1491-1498, 1999.

[30] R. Moeller, R. Horejsi, S. Pilz et al., "Evaluation of risk profiles by subcutaneous adipose tissue topography in obese juveniles," Obesity, vol. 15, no. 5, pp. 1319-1324, 2007.

[31] B. R. Winkelmann, W. März, B. O. Boehm et al., "Rationale and design of the LURIC study-a resource for functional genomics, pharmacogenomics and long-term prognosis of cardiovascular disease," Pharmacogenomics, vol. 2, supplement 1, pp. S1-S73, 2001.

[32] P. L. van Haelst, A. Liem, A. J. van Boven et al., "Usefulness of elevated neopterin and C-reactive protein levels in predicting cardiovascular events in patients with non-Q-wave myocardial infarction," American Journal of Cardiology, vol. 92, no. 10, pp. 1201-1203, 2003.

[33] P. Cirillo, M. Pacileo, S. De Rosa et al., "Neopterin induces proatherothrombotic phenotype in human coronary endothelial cells," Journal of Thrombosis and Haemostasis, vol. 4, no. 10, pp. 2248-2255, 2006.

[34] J. Auer, R. Berent, E. Laßnig, and B. Eber, "Serum neopterin and activity of coronary artery disease," Heart Disease, vol. 3, no. 5, pp. 297-301, 2001.

[35] G. Hoffmann, W. Schobersberger, S. Frede et al., "Neopterin activates transcription factor nuclear factor $-\kappa \mathrm{B}$ in vascular smooth muscle cells," FEBS Letters, vol. 391, no. 1-2, pp. 181$184,1996$.

[36] D. Fuchs, P. Avanzas, R. Arroyo-Espliguero, M. Jenny, L. Consuegra-Sanchez, and J. C. Kaski, "The role of neopterin in atherogenesis and cardiovascular risk assessment," Current Medicinal Chemistry, vol. 16, no. 35, pp. 4644-4653, 2009.

[37] G. Weiss, C. Murr, H. Zoller et al., "Modulation of neopterin formation and tryptophan degradation by Th1- and Th2derived cytokines in human monocytic cells," Clinical and Experimental Immunology, vol. 116, no. 3, pp. 435-440, 1999.

[38] P. Mayersbach, D. Fuchs, and H. Schennach, "Performance of a fully automated quantitative neopterin measurement assay in a routine voluntary blood donation setting," Clinical Chemistry and Laboratory Medicine, vol. 48, no. 3, pp. 373377, 2010

[39] M. Jenny, M. Klieber, D. Zaknun et al., "In vitro testing for anti-inflammatory properties of compounds employing peripheral blood mononuclear cells freshly isolated from healthy donors," Inflammation Research. In press.

[40] H. Sprenger, C. Jacobs, M. Nain et al., "Enhanced release of cytokines, interleukin-2 receptors, and neopterin after longdistance running," Clinical Immunology and Immunopathology, vol. 63, no. 2, pp. 188-195, 1992. 
[41] G. P. Tilz, W. Domej, A. Diez-Ruiz et al., "Increased immune activation during and after physical excercise," Immunobiology, vol. 188, no. 1-2, pp. 194-202, 1993.

[42] M. Ristow, K. Zarse, A. Oberbach et al., "Antioxidants prevent health-promoting effects of physical exercise in humans," Proceedings of the National Academy of Sciences of the United States of America, vol. 106, no. 21, pp. 8665-8670, 2009.

[43] J. Sun, G. K. Sukhova, P. J. Wolters et al., "Mast cells promote atherosclerosis by releasing proinflammatory cytokines," Nature Medicine, vol. 13, no. 6, pp. 719-724, 2007. 


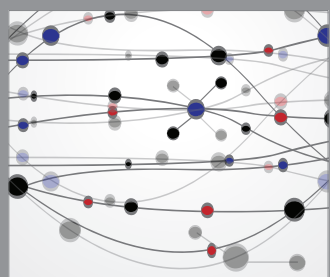

The Scientific World Journal
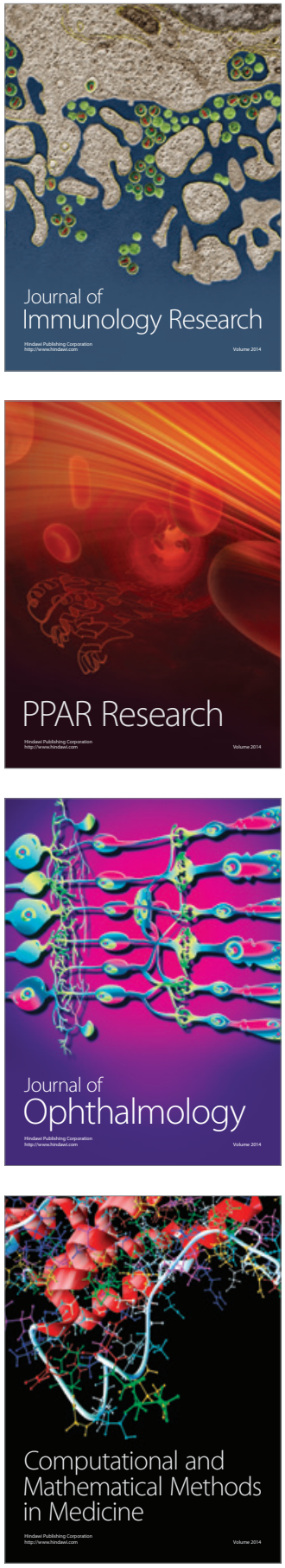

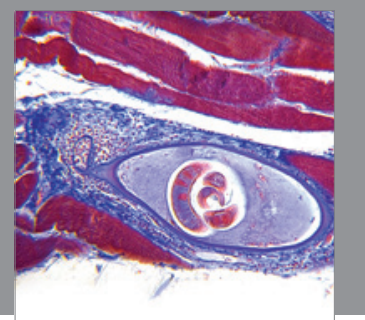

Gastroenterology

Research and Practice
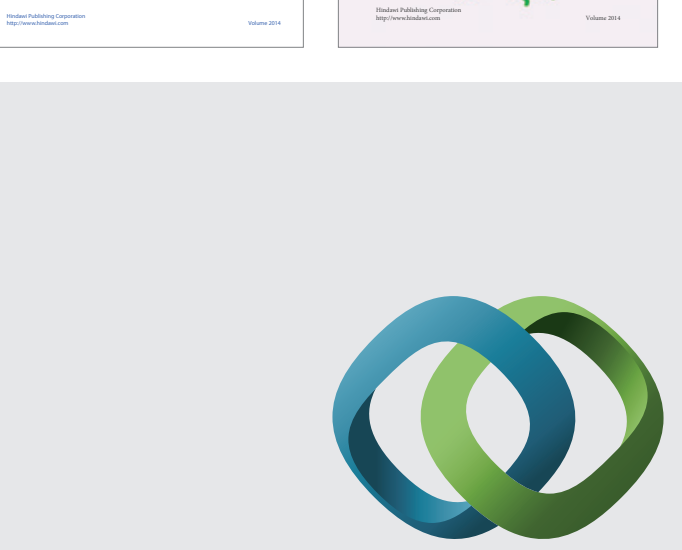

\section{Hindawi}

Submit your manuscripts at

http://www.hindawi.com
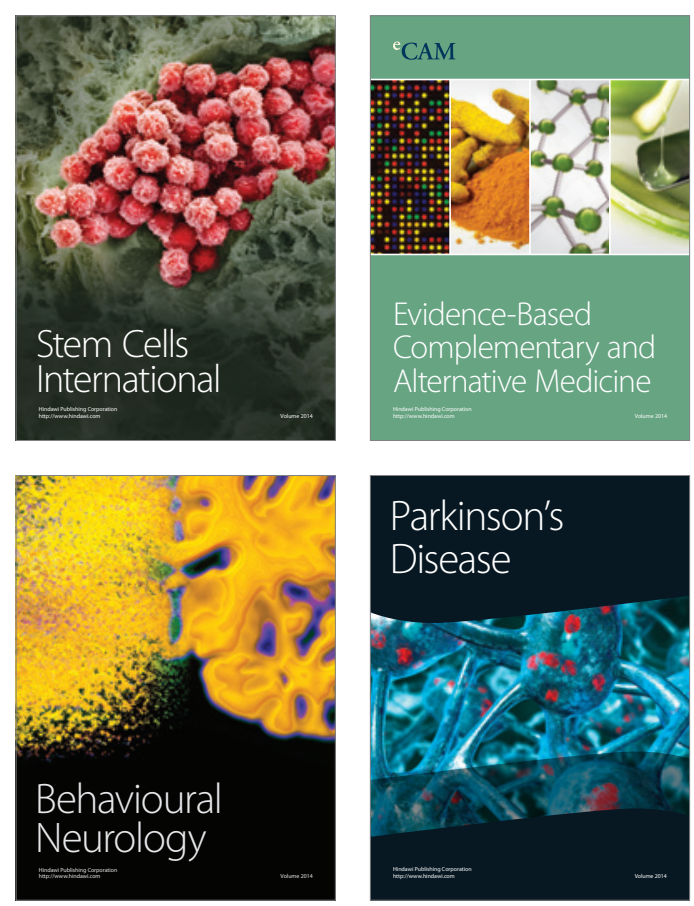

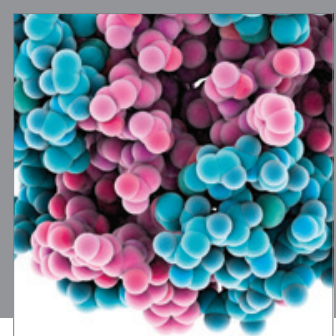

Journal of
Diabetes Research

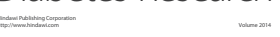

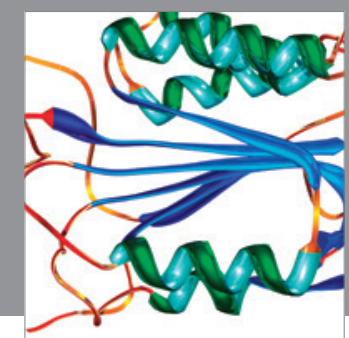

Disease Markers
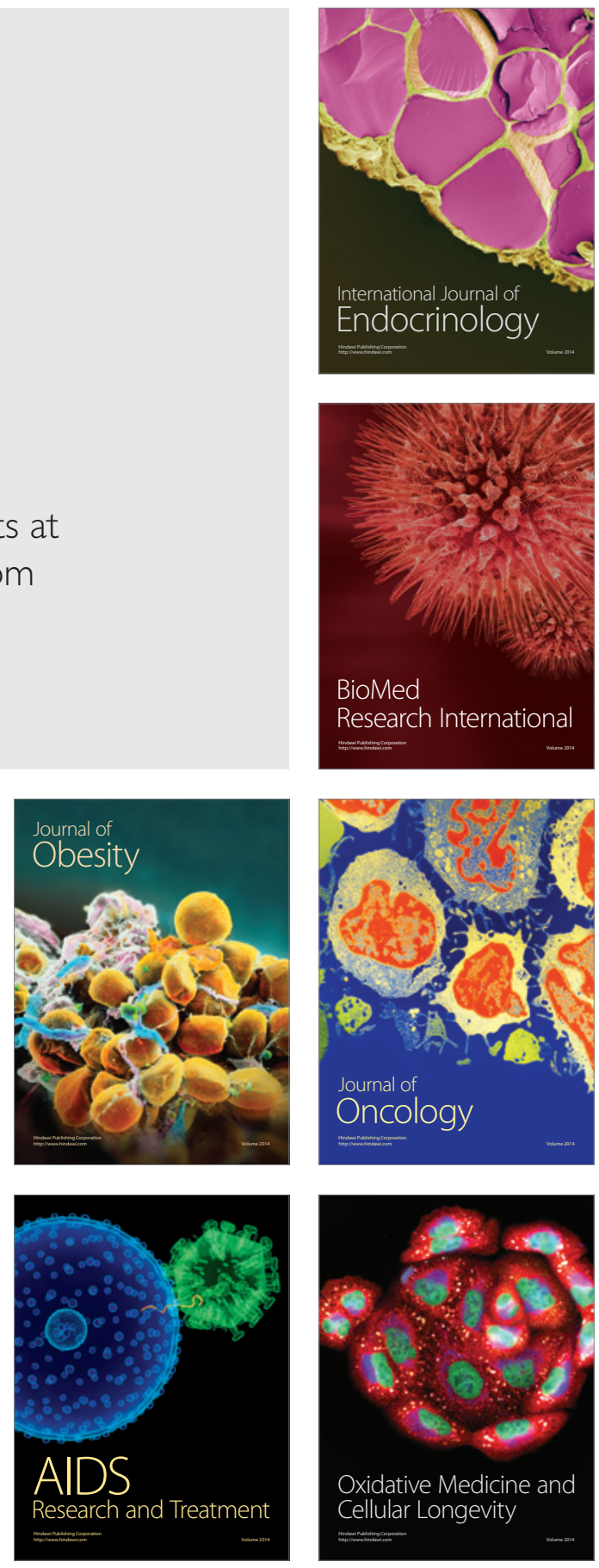